\title{
Rechtsgeschichte
}

\section{Christoph Schönberger}

\section{Wissenschaftsgeschichte als Schlüssel zur Geschichte des öffentlichen Rechts?}

Bemerkungen zu einem schwierigen Verhältnis 


\section{Wissenschaftsgeschichte als Schlüssel zur Geschichte des öffentlichen Rechts?}

Bemerkungen zu einem schwierigen Verhältnis

Die wichtigste Leistung von Michael Stolleis ist die Etablierung der Wissenschaftsgeschichte des öffentlichen Rechts als wissenschaftliche Disziplin. Sie manifestiert sich vor allem in seiner umfassenden "Geschichte des öffentlichen Rechts in Deutschland " ${ }^{\mathbf{I}}$ deren vierter, das Nachkriegsdeutschland bis zur Wiedervereinigung behandelnder Band vor dem Abschluss steht. Diese Leistung ist umso bemerkenswerter, als sie gegenläufigen Fachtraditionen abgerungen werden musste. Rechtsgeschichtliche Lehrstühle sind in Deutschland herkömmlich im Bereich des Privatrechts angesiedelt. Im öffentlichen Recht hat sich insoweit allein die Verfassungsgeschichte etwas ausdifferenziert. Der Geschichte des öffentlichen Rechts wie auch der Strafrechtsgeschichte fehlt weitgehend das institutionelle Fundament in den juristischen Fakultäten, und die zunehmende Erosion der Grundlagenfächer insgesamt verstärkt diese traditionelle Schwäche in unseren Tagen eher noch. Stolleis' Leistung nimmt sich vor diesem Hintergrund besonders beeindruckend aus. Gleichwohl wirft gerade die Form, in der er sich der Geschichte des öffentlichen Rechts genähert hat, spezifische Fragen auf, die zur Klärung der Grundprobleme der rechtsgeschichtlichen Forschung auf dem Gebiet des öffentlichen Rechts beitragen können.

I.

Das zentrale Problem ist dabei das Verhältnis der Geschichte des öffentlichen Rechts zu dessen Wissenschaftsgeschichte. Es zeigt sich schon in der Benennung von Stolleis' bereits klassischen Bänden. Sie führen die "Geschichte des öffentlichen Rechts « im Titel, behandeln aber nicht eigentlich die Geschichte des öffentlichen Rechts im Sinne einer Geschichte seiner Praxis durch Rechtsetzung, Verwaltung und Gerichte. Vielmehr konzentriert Stolleis seine Aufmerksamkeit ganz auf die Geschichte der Wissenschaft

\footnotetext{
I Michael Stolleis, Geschichte des öffentlichen Rechts in Deutschland, Bd. I: Reichspublizistik und Policeywissenschaft I600-I 800, München I988; Bd. 2: Staatsrechtslehre und Verwaltungswissenschaft $\mathrm{I} 800-$ I9I4, München I992; Bd. 3: Staats- und Verwaltungsrechtswissenschaft in Republik und Diktatur 19I4-I945, München I999.
} 
vom öffentlichen Recht »als Literaturgeschichte der wissenschaftlichen Erfassung, der dogmatischen Durchdringung und Systematisierung des öffentlichen Rechts «. ${ }^{2}$ Im Kern geht es ihm um die intellektuelle Produktion an den deutschen Universitäten, um »Theoriebildung und deren pädagogische Vermittlung «. ${ }^{3}$ Die Grundorientierung ist damit klar: Im Zentrum der Darstellung stehen die wissenschaftlichen Schriften deutscher Professoren im Bereich des öffentlichen Rechts. Insoweit diese Geschichtsschreibung sich auf Institutionen und deren Praxis bezieht, geht es nicht um Verfassungen, Verwaltungen und Gerichte, sondern wie selbstverständlich um die Universitäten, um Fakultäten, Fächerzuschnitte, Vorlesungen, Studien- und Prüfungsordnungen. Ohne Ideengeschichte im Sinne eines fragwürdig gewordenen methodischen Idealismus zu sein, ist diese Wissenschaftsgeschichte eine »intellectual history «, die diskurs-, mentalitäts- und universitätsgeschichtlich eingebettet ist. ${ }^{4}$ Bereits eine derartige Wissenschaftsgeschichte stößt auf erhebliche Herausforderungen und Schwierigkeiten, etwa hinsichtlich der zeitlichen, räumlichen und gegenständlichen Eingrenzung des Stoffs. ${ }^{5}$ Insoweit sie wie bei Stolleis eine enzyklopädische Darstellungsform wählt, ist sie überdies unvermeidlich mit der Frage konfrontiert, welchen Raum sie den inhaltlichen Auseinandersetzungen gegenüber den Ausführungen zu Territorien, Universitäten und Personen gibt. Diesen Problemen kann hier nicht näher nachgegangen werden. Vielmehr soll es um die allgemeinere Frage gehen, wie sich die Wissenschaftsgeschichte des öffentlichen Rechts zur Geschichte des öffentlichen Rechts verhält.

II.

Kann man die Wissenschaftsgeschichte des öffentlichen Rechts ohne die Geschichte des öffentlichen Rechts schreiben? Was erfährt man aus der einen über die andere? Die Geschichte des öffentlichen Rechts ist in erster Linie die Geschichte einer normativ angeleiteten Praxis. Es geht um Verfassungen und Gesetze, um Verwaltungen und Gerichte, um alltägliches Handeln von Amtsträgern, das rechtlich ermöglicht, begrenzt oder legitimiert wird. Die Wissenschaftsgeschichte nimmt hingegen nicht diese Praxis in den Blick, sondern die wissenschaftliche Produktion auf dem Themenfeld des öffentlichen Rechts. Die Publikationen mögen sich direkt oder indirekt auf die Praxis des öffentlichen Rechts bezogen haben, sie

2 Stolleis, Geschichte (Fn. I), Bd. I, 43 .

3 Stolleis, Geschichte (Fn. I), Bd. I, 44.

4 Stolleis, Geschichte (Fn. I), Bd. I, $45 \mathrm{f}$.

5 Stolleis, Geschichte (Fn. I), Bd. I, $46 \mathrm{ff}$. 
werden indes zunächst einmal um ihrer selbst willen untersucht. Man will wissen, was an deutschen Universitäten in der Vergangenheit über das öffentliche Recht gedacht und geschrieben wurde.

Das führt freilich sofort in ein Dilemma hinein. Denn es geht um die Wissenschaftsgeschichte einer Disziplin, die in gewissem Ausmaß immer auf eine Praxis bezogen war. Lässt sich die Historie der Disziplin dann aber überhaupt schreiben, ohne dass zugleich diese Praxis in den Blick genommen wird? Hierbei handelt es sich um ein allgemeines Problem der Wissenschaftsgeschichte aller in spezifischer Weise praktischen Universitätsdisziplinen. Die naheliegende Vergleichsdisziplin wäre wohl die Medizin, die schon in den mittelalterlichen Universitäten die eigentliche praktische Schwesterwissenschaft der Jurisprudenz bildete. Die Medizingeschichte lässt sich wahrscheinlich nicht allein als Geschichte der wissenschaftlichen Produktion von Medizinprofessoren schreiben. Sie verlangt geradezu danach, um eine Geschichte der medizinischen Praxis ergänzt zu werden. Waren die wissenschaftlichen Traktate einflussreich? Haben Sie die Praxis beeinflusst? Wurde aufgrund entsprechender Theoreme behandelt, operiert, geheilt? Insoweit stellt sich die Problematik der Wissenschaftsgeschichte stärker praktischer Disziplinen anders dar als diejenige von Wissenschaftsgebieten, denen keine soziale Praxis unmittelbar entspricht. Die Wissenschaftsgeschichte der Historiographie beispielsweise lässt sich sicherlich als eigenständige intellectual history schreiben. Zwar ist auch hier etwa interessant, ob die Werke ein gesellschaftliches Echo fanden oder breiteren Strömungen Ausdruck verliehen. Aber es fehlt doch eine strukturierte gesellschaftliche Praxis, für welche die entsprechende Universitätsdisziplin ausbildet und mit welcher diese auch über diese Ausbildungsleistung hinaus in irgendeiner Form verknüpft ist.

Für die Wissenschaftsgeschichte des öffentlichen Rechts liegen die Dinge von vornherein anders. Man hat es hier nie mit einer völlig autonomen Disziplin zu tun, die ihre wissenschaftlichen Produkte ohne Rücksicht auf die juristische Praxis entwirft. Es laufen vielmehr vielfältige Verbindungslinien zwischen dieser Praxis und der wissenschaftlichen Theoriebildung hin und her. Vor diesem Hintergrund scheint klar, dass es keine Wissenschaftsgeschichte des öffentlichen Rechts geben kann, die von dieser Praxisverflechtung ganz absehen dürfte. Man bekäme dann einen Torso und verlöre die Eigenart rechtswissenschaftlicher Produktion 
von vornherein aus dem Blick. Sicherlich war dieser Praxisbezug je nach Epoche oder wissenschaftlicher Literaturgattung stark oder eher schwach ausgeprägt. Ein verwaltungsrechtlicher Kommentar hatte einen stärkeren Praxisbezug als ein staatstheoretischer Traktat. Die unterschiedliche Intensität des Praxisbezugs hängt auch mit der Frage zusammen, welche Institutionen für die Beantwortung von Rechtsfragen auf dem jeweiligen Gebiet überhaupt maßgeblich waren. Hier bestand historisch stets eine große Variationsbreite. Paul Labands "Staatsrecht des Deutschen Reiches « systematisierte etwa im Deutschland Bismarcks und Wilhelms II. ein Verfassungsrecht, dessen Anwendung weitgehend bei den politischen Institutionen lag. Will man den oft behaupteten kanonischen Status des Werks genauer untersuchen, dann bedarf es eingehender Quellenforschung im Hinblick auf die staatsrechtliche Praxis in den Institutionen und Behörden des Deutschen Kaiserreichs.

Akzeptiert man, dass es eine gegenüber der Rechtspraxis isolierte Wissenschaftsgeschichtsschreibung des öffentlichen Rechts nicht geben kann - und auch Stolleis redet einer derartigen Wissenschaftsgeschichtsschreibung selbstverständlich nicht das Wort -, dann drängt sich freilich ein Folgeproblem auf. Es geht dann um die Frage, wie die Wissenschaftsgeschichte diesen Praxisbezug berücksichtigen und verarbeiten kann. Die naheliegendste Form einer derartigen Verarbeitung versteht die Wissenschaftsgeschichte des öffentlichen Rechts als Abkürzung zu dessen Geschichte überhaupt. Sie versucht, über die Wissenschaftsgeschichte die Geschichte des Rechts selbst zu erschließen. Dieser Weg ist sowohl praktisch wie theoretisch verführerisch. In praktischer Hinsicht reduziert sich dadurch das den Historiker stets plagende Quellenproblem: Auch wenn seit der frühen Neuzeit eine große Fülle gedruckter Quellen aus der Wissenschaftsgeschichte vorliegt, ist der entsprechende Bestand doch deutlich überschaubarer als die möglichen Quellen für die gesamte Rechtspraxis in Form von Verwaltungs- und Gerichtsakten oder parlamentarischen Verhandlungen, zumal im vielschichtig-föderativen Deutschland. Auch in theoretischer Hinsicht scheint dieser Weg ansprechend. Man lehnt sich dadurch an die Versuche in der Zeit an, das öffentliche Recht in eine gewisse sprachliche und systematische Form der Darstellung zu bringen. Man profitiert von den begrifflichen Anstrengungen der wissenschaftlichen Zeitgenossen und nutzt die Angebote, die an den Uni- 
versitäten gewissermaßen à chaud für das öffentliche Recht der jeweiligen Epoche entwickelt wurden.

Allerdings stellt sich für diesen Ansatz das Problem, wie zu beurteilen ist, ob denn die wissenschaftliche Produktion tatsächlich für die zeitgenössische Praxis des öffentlichen Rechts relevant oder doch wenigstens vielleicht repräsentativ war. Die Gefahr des Ansatzes liegt nicht so sehr in einem möglichen Rückfall in eine pure Ideengeschichte älterer Prägung. Er wird aber vielleicht allzu rasch dazu neigen, die wissenschaftliche Literatur als getreue Widerspiegelung der rechtlichen Zustände der jeweiligen Epoche zu verstehen.

Nun ließe sich dem entgegnen, dass die Wissenschaftsgeschichte immer nur cum grano salis als Abkürzung zur Geschichte des Rechts verwendet werden dürfe. Denn es gehe ja eben in erster Linie nur um Wissenschaftsgeschichte und nicht gleichzeitig auch noch umfassend um die Geschichte des Rechts selbst. Weder beanspruche die Wissenschaftsgeschichte, die Entwicklung des Rechts selbst zu beschreiben, noch dürfe der Leser einer derartigen Geschichte diese Erwartung an ein derartiges Werk richten. Dieses Argument hilft aber nicht recht weiter. Wenn die Wissenschaftsgeschichte einer praktischen Disziplin nicht ohne Blick auf die Praxis geschrieben werden kann, dann muss sie eine Form entwickeln, den Leser über die Wechselbezüge zu dieser Praxis zu orientieren. In Stolleis' Wissenschaftsgeschichte geschieht das denn auch immer wieder nebenbei wie selbstverständlich. So wird der Leser etwa in den Passagen zur Herausbildung der Verwaltungsrechtswissenschaft darüber informiert, dass deren Ausbau erst durch die Einführung der Verwaltungsgerichtsbarkeit möglich wurde. ${ }^{6}$ Das Wechselverhältnis von Wissenschaft und Praxis tritt hervor: "Auf diese Weise beeinflussten sich Verwaltungsalltag, Gesetzgebung, Rechtsprechung und Wissenschaft gegenseitig und brachten das Fach an verschiedenen Stellen gleichzeitig hervor, in der rechtsgebundenen Verwaltungstätigkeit, im Urteilsspruch, in Gesetzeskommentar und Monographie sowie in der Lehre, die freilich erst im 20. Jahrhundert ihr (studentisches) Publikum wirklich erreichte. «7

Die Frage ist also nicht, ob diese Wechselbezüge erörtert werden, sondern in welcher Form dies geschieht, auf welche Quellen dabei Bezug genommen wird und wie diese Verarbeitung methodisch reflektiert wird. War etwa Otto Mayers Lehrbuch des deut-

6 Stolleis, Geschichte (Fn. I), Bd. 2, $242 \mathrm{f}$.

7 Stolleis, Geschichte (Fn. I), Bd. 2, 243. 
schen Verwaltungsrechts für die Praxis der (Länder-)Verwaltungen im kaiserlichen Deutschland wirklich von Bedeutung? Das ist keineswegs geklärt, und die rasche hohe Anerkennung für Mayers Buch in der zeitgenössischen wissenschaftlichen Diskussion ${ }^{8}$ kann diese Frage nicht beantworten. Dass er mit einigen seiner Positionen - wie etwa dem Versuch, den französischen »domaine public « als Lehre vom öffentlichen Eigentum in Deutschland heimisch zu machen ${ }^{9}$ - selbst in der wissenschaftlichen Debatte nicht durchdrang, könnte bereits ein Hinweis sein, dass die Rezeption seiner Lehren auch in der Praxis keineswegs selbstverständlich war. Es ist nicht ausgeschlossen, dass man aus der Lektüre der Entscheidungen des preußischen Oberverwaltungsgerichts mehr und anderes über die Geschichte des Verwaltungsrechts im deutschen Kaiserreich erfährt als aus derjenigen von Otto Mayers klassischem Lehrbuch. Natürlich sind derartige Fragen oft außerordentlich schwer zu beantworten, und häufig wird sogar unklar sein, welche Quellen man untersuchen müsste, um entsprechende Antworten zu finden. Wichtig für die Wissenschaftsgeschichtsschreibung scheint aber zunächst einmal die Erkenntnis zu sein, dass das häufige Wechselverhältnis von Wissenschaft und Praxis nicht so verstanden werden kann, dass sich letztlich doch immer an der Wissenschaft die Praxis ablesen lässt.

III.

Die Einbeziehung des Praxisbezugs der Disziplin in die Wissenschaftsgeschichte des öffentlichen Rechts bedarf vor diesem Hintergrund einer stärker expliziten Reflexion auf die spezifischen institutionellen und rechtskulturellen Bedingungen, unter denen die jeweilige rechtswissenschaftliche Produktion ablief. Implizite Annahmen über die Aussagefähigkeit wissenschaftsgeschichtlicher Erkenntnisse für die Geschichte des öffentlichen Rechts gehören dabei generell stärker auf den Prüfstand. Es ist möglich, dass die in Deutschland bis heute besonders enge Wechselbeziehung von Wissenschaft und Praxis der deutschen rechtsgeschichtlichen Diskussion den Blick auf das Problem eher verstellt. Angesichts des vielfältigen Austauschs zwischen Rechtswissenschaft und Rechtspraxis ist es in Deutschland vielleicht leichter als anderswo möglich, von der Wissenschaftsgeschichte auf die Geschichte des öffentlichen Rechts zu schließen. Gerade diese Hypothese bedürfte aber

8 Stolleis, Geschichte (Fn. I), Bd. 2, 407.

9 Stolleis, Geschichte (Fn. I), Bd. 2, 4I3. 
einer genaueren Reflexion auf die spezifischen Bedingungen dieser Verflechtung in der deutschen Rechtstradition und auf die Aussagekraft, die wissenschaftsgeschichtlichen Einsichten aufgrund dieser Wechselwirkungen für die Geschichte des öffentlichen Rechts selbst zukommen kann.

Hier dürfte die gerade auch von Michael Stolleis angemahnte vergleichende Wissenschaftsgeschichte des öffentlichen Rechts, ${ }^{\text {Io }}$ die leider immer noch in den Kinderschuhen steckt, ${ }^{\text {II }}$ aufschlussreiche Erkenntnisse liefern können. Bereits ein kursorischer vergleichender Blick in der Gegenwart lehrt nämlich, dass die besonders enge Verflechtung von akademischer Rechtswissenschaft und Rechtspraxis in Deutschland ein Sonderphänomen ist, das in den europäischen Nachbarländern nach Art und Intensität kaum eine Entsprechung findet. Der Verfasser ist darauf in jüngster Zeit durch die Mitarbeit an dem Handbuch »Ius Publicum Europaeum « immer wieder neu aufmerksam worden. Wer etwa die französische Rechtswissenschaft und Rechtspraxis auf dem Gebiet des öffentlichen Rechts kennt, kann kaum umhin zu beobachten, dass die französische Rechtswissenschaft insgesamt wesentlich praxisferner ist als die deutsche und von der Rechtspraxis auch viel weniger wahrgenommen wird. Die Gründe dafür reichen von der schwächeren Stellung der Universitäten im Allgemeinbewusstsein - an der Spitze des Bildungssystems stehen nicht die Universitäten, sondern die "grandes écoles « - über das Fehlen einer einheitlichen Juristenausbildung bis hin zu der Tatsache, dass die französische Verwaltung anders als die deutsche traditionell nicht in erster Linie eine Juristenverwaltung ist. Sicherlich hat diese Situation in der Vergangenheit nicht immer in einer derart ausgeprägten Weise bestanden. Eine Wissenschaftsgeschichte des französischen öffentlichen Rechts - sie ist auffälligerweise weitgehend ungeschrieben, während die Geschichte der französischen Verwaltung seit Pierre Legendre $^{\mathbf{I 2}}$ immer wieder untersucht wurde - stünde aber jedenfalls von vornherein viel stärker vor dem Problem, die Distanz und das vergleichsweise geringe Gewicht der »doctrine « gegenüber der Rechtspraxis zu verarbeiten. Die Selbstverständlichkeit, mit der Michael Stolleis sich für Deutschland auf die Wissenschaftsgeschichte des öffentlichen Rechts als eigenständigen Gegenstand konzentrieren konnte, ist für Frankreich kaum vorstellbar.

Das bedeutet aber im Kontrast auch, dass es für Deutschland einer sehr viel genaueren Analyse der Gründe für die hierzulande so

Io Stolleis, Geschichte (Fn. I), Bd. 2, $6 \mathrm{f}$.

I I Was hier in monographischer Form bereits möglich ist, zeigt besonders eindrucksvoll die gemeineuropäische Verwaltungsrechtsgeschichte von L. MANNORI, B. SoRDI, Storia del diritto amministrativo, Rom, Bari 2006.

I 2 P. Legendre, Trésor historique de l'État en France. L'Administration classique (I968), 2. Aufl. Paris

I992. 
ausgeprägte Sonderstellung der akademischen Rechtswissenschaft und ihre spezifische Verflechtung mit der Rechtspraxis bedarf. ${ }^{\mathrm{I}}$ Hier spielt sicherlich die besondere Rolle des Rechts und der juristischen Fakultäten als Träger eines gewissen Einheitsbewusstseins in einem vielfältig zerklüfteten und erst spät national geeinten politischen Gebilde eine bedeutsame Rolle. Gerade für das öffentliche Recht ist nicht zu übersehen, wie sehr es gerade die Wissenschaft war, die über die Partikularität der vielfältigen Staats- und Verwaltungsrechtssysteme der Einzelstaaten hinweg überhaupt erst eine gewisse systematische Einheit dieses Rechtsgebiets hervorbrachte. Noch Otto Mayers am einheitsstaatlichen Frankreich orientierte Abstraktionen waren vor allem auch Ausdruck des Versuchs, die Heterogenität der einzelstaatlichen Verwaltungsrechtsordnungen im deutschen Kaiserreich ein Stück weit zu transzendieren. ${ }^{\mathbf{I}}$ In den häufig kleinstaatlichen deutschen Verhältnissen stellten die juristischen Fakultäten der jeweiligen Landesuniversitäten überdies von vornherein eine auch für die Rechtspraxis dringend benötigte Elite dar. Die besondere Rolle professoraler Gutachten in der deutschen Rechtspraxis - für die es international wiederum keine Entsprechung gibt - setzt heute sicherlich noch in gewisser Weise die ältere Praxis der Aktenversendung an die Fakultäten fort. Der hohe Professorenanteil bei der Besetzung des Bundesverfassungsgerichts lässt sich ebenfalls als Ausdruck derartiger Traditionslinien verstehen.

Michael Stolleis hat auf solche Zusammenhänge immer wieder hingewiesen. In der Einleitung zum ersten Band seiner Wissenschaftsgeschichte des öffentlichen Rechts »in Deutschland « hob er I 988 - im noch geteilten Land, dessen nahe Wiedervereinigung kaum jemand vorausahnte - besonders hervor, dass sein Interesse an der Wissenschaftsgeschichte des Alten Reiches mit der schwierigen Frage nach der deutschen Identität zusammenhing, und es schwang dabei eine gewisse Rehabilitierung des rechtsbasierten Reichszusammenhangs gegenüber der nationalstaatlichen Historiographie des I9. Jahrhunderts mit: ${ }^{15}$ " Gerade wegen der notorischen Partikularität und Zerrissenheit, die immer wieder praktisch erlitten wurden - so sehr man ihre Vorteile als Friedensordnung und Nährboden kultureller Vielfalt pries -, wurde in Deutschland die Suche nach einem die Teilung überwindenden Naturrecht, nach einem ius publicum universale oder sallgemeinen Staatsrecht be- $^{-}$ sonders intensiv betrieben." Es mag aber sein, dass Stolleis gele-

I3 Eine Fülle anregender historischvergleichender Beobachtungen dazu finden sich bereits bei J.P. DAwson, The oracles of the law, Ann Arbor 1968, S. I48 ff.

I4 Chr. SChönberger, Verwaltungsrechtsvergleichung: Eigenheiten, Methoden und Geschichte, in: v. Bogdandy / CASSESE / HuBER (Hg.), Ius Publicum Europaeum, Bd. IV, Heidelberg 20I I, \$ 7I, Rdnr. $38 \mathrm{f}$.
I5 Stolleis, Geschichte (Fn. I), Bd. I, $55 \mathrm{f}$. 
gentlich unterschätzt hat, wie spezifisch deutsch die entsprechende Sonderstellung der Rechtswissenschaft ist und wie sehr sie gerade deshalb in ihrer Eigenart auch und gerade in einer Wissenschaftsgeschichte immer wieder explizit zum Thema gemacht werden muss. In diesem Sinne ist eine auf Universitäten und Gelehrte konzentrierte Wissenschaftsgeschichte des öffentlichen Rechts vielleicht selbst ein sehr deutsches Unternehmen. Das stellt das Unternehmen nicht infrage, kennzeichnet es aber doch in seiner Eigenart und seinem spezifischen Zuschnitt. Und es ist wohl auch mehr als nur ein glücklicher biographischer Zufall, dass Michael Stolleis den wissenschaftsgeschichtlichen Weg vom Alten Reich zum Deutschland des 20. Jahrhunderts in dem Zeitraum durchschritten hat, in dem das geteilte Land seinen Weg zum Gleichklang von deutscher und europäischer Einigung fand.

Christoph Schönberger 